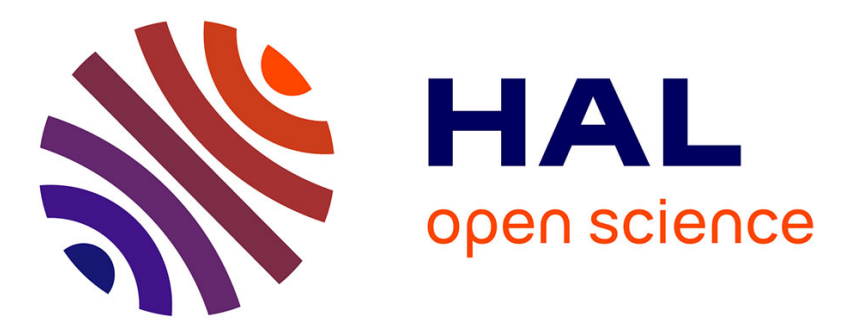

\title{
A Prioritized Multi-Channel Multi-Time slot MAC Protocol For Large-Scale Wireless Sensor Networks
} Jamila Ben Sliman, Ye-Qiong Song, Anis Koubâa

\section{To cite this version:}

Jamila Ben Sliman, Ye-Qiong Song, Anis Koubâa. A Prioritized Multi-Channel Multi-Time slot MAC Protocol For Large-Scale Wireless Sensor Networks. ComNet'09: The First International Conference on Communications, and Networking, Nov 2009, Hammamet, Tunisia. pp.8. inria-00435517

\section{HAL Id: inria-00435517 https://hal.inria.fr/inria-00435517}

Submitted on 24 Nov 2009

HAL is a multi-disciplinary open access archive for the deposit and dissemination of scientific research documents, whether they are published or not. The documents may come from teaching and research institutions in France or abroad, or from public or private research centers.
L'archive ouverte pluridisciplinaire $\mathbf{H A L}$, est destinée au dépôt et à la diffusion de documents scientifiques de niveau recherche, publiés ou non, émanant des établissements d'enseignement et de recherche français ou étrangers, des laboratoires publics ou privés. 


\title{
A Prioritized Multi-Channel Multi-Time slot MAC Protocol For Large-Scale Wireless Sensor Networks
}

\author{
Jamila Ben Slimane* ${ }^{* \ddagger}$ Ye-Qiong Song ${ }^{\ddagger}$ and Anis Koubaa ${ }^{\S \nsubseteq}$ \\ *Sup'Com-MEDIATRON, City of Communication Technologies, 2083 Ariana, Tunisia \\ $\ddagger$ LORIA and INPL, Campus Scientifique, BP 23954506 Vandoeuvre-les-Nancy, France \\ §IPP-HURRAY! Research Group, Polytechnic Institute of Porto, \\ Rua Antnio Bernardino de Almeida, 431, 4200-072 Porto, Portugal \\ 『Al-Imam Muhammad ibn Saud University, Computer Science Dept., 11681 Riyadh, Saudi Arabia \\ Email: jamilabs07@yahoo.fr, Song@loria.fr, akoubaa@dei.isep.ipp.pt
}

\begin{abstract}
This paper addresses a new prioritized multichannel multi-time slot MAC protocol (PMCMTP) for large-scale WSNs especially for Ultra-Wide Band (UWB) based networks. To reduce the complexity of resource sharing, the global network is composed of a set of Personal Area Networks (PANs) or cells. According to available resource and PANs duty cycle, PMCMTP can dynamically assign several data channels per PAN and efficiently allocate time slots to each PAN's members. This significantly decreases delay and increases throughput. Through some simulations, we evaluate the performance of the proposed protocol. The results show that PMCMTP ensures an efficient and fair channels allocation between cells permitting, on the one hand, an enhancement of quality-of-service inside each PAN and, on the other hand, a maximization of channel utility.
\end{abstract}

\section{INTRODUCTION}

Wireless sensor networks may consist of a collection of spatially distributed autonomous sensor nodes characterized by limited memory, processing capability and battery power supply. Such networks represent an emerging technology with wide range of potential applications such as medical systems, environment monitoring, military applications, etc. An important fact that can not be neglected in WSNs is the need of quality-of-service (QoS) support. According to novel application requirements, QoS constraints become more and more critical in terms of end-to-end delay and data throughput. Also, due to energetic constraints at node level, energy saving remains the most challenging issue. Both IEEE 802.15.4 and its recent amendment IEEE 802.15.4a standards allow dynamic channel allocation and use of multiple channels available at their physical layers but its MAC protocols are designed only for single channel. Also, sensor's transceivers such as CC2420 used by current WSN hardware (MICAZ, TelosB, and CMU FireFly), provide multiple channels and as shown in [1] and [2] channel switch latency of CC2420 transceiver is short (just about $200 \mu \mathrm{s}$ ). However, in the general case of dense Mesh WSNs, the exploitation of the multichannel access is complex. This explains the imperative need to propose an adequate network architecture that can simplify and reduce the complexity of the resource sharing task in such networks and to design a scalable and optimal multi-frequency MAC protocols. Multi-channel Media Access Control (MAC) protocols should allow parallel transmissions with efficient use of available resource (channels and time slots), without interference, data communication conflict and control packet overhead. Most currently deployed sensor networks use the same channel to communicate information towards nodes. This is a source of great inefficiency as it poorly utilizes the available wireless spectrum. This paper takes advantage of radio communication capabilities of IEEE 802.15.4a IRUWB compliant devices that benefit from UWB technology advantages as specified in the IEEE 802.15.4a standard and that can communicate on multiple frequencies as specified in the IEEE 802.15.4 standard.

The main problem addressed is designing an efficient prioritized multi-channel and multi-time slot allocation MAC protocol for dense and large-scale WSNs based principally on the IEEE 802.15.4a IR-UWB physical layer. Our paper makes the following main contributions:

- First, focusing on UWB technology, it provides the first spectrum management scheme exclusively for full mesh Largescale WSNs.

- Second, it proposes PMCMTP, a Prioritized Multi-Channel Multi-Time slot media access control Protocol for dense and large-scale WSNs to ensure an efficient resource allocation, in terms of channel frequencies and time slots inside each PAN, obeying to QoS constraints (i.e priority of resource requests). PMCMTP takes into account: the spatial channel reuse, the duty cycle's information of PANs and the support of data stream prioritization.

- Finally, using simulations, we perform an evaluation of our protocol, demonstrating that it comes to reach our goals in terms of spectrum efficiency and network performance enhancement.

\section{A. Related Works}

Designing a good MAC protocol represents one of the most challenging tasks that enable the successful operation of the network and the improvement of its performance. The majority of WSNs use a single channel for control and data traffic what leads to a complex problem of resource sharing especially in dense and large scale networks. Although multi-channel access protocols for WSNs are not new issues and have been a topic of research in such networks, proposed protocols are really 
very few. The principle of frequency allocation is firstly used in cellular networks like Global System for Mobile communications (GSM) [3]. In GSM networks, channel allocation is a function of network sizing (taking into account traffic distribution and subscriber model) and cellular planning, so the minimum distance of frequency reuse $D=\sqrt{3 K} \times R$ where $R$ represents the cell's radius and $K$ represents the cluster's scale. However in WSNs, frequency reuse is a function of sensor's coverage, duty cycle of network's members and network's topology. In the literature, a number of multichannel MAC protocols have been proposed for WSNs [4]-[8]. The first multi-channel protocol, called Multi-frequency Media access control for wireless Sensor Networks (MMSN) [4], represents four frequency assignment schemes for WSN: exclusive frequency assignment, even selection, eavesdropping and implicit-consensus. The first scheme is used when the number of available frequencies is at least as large as the two-hop node number, it allocates channels in such a way that nodes within two hops are assigned different frequencies. Due to several broadcasts, the communication overhead in this scheme is relatively high. The second scheme ensures smaller overhead but it requires more physical frequencies that is, it assumes that frequency resource are abundant. Given that the two other schemes do not guarantee the assignment of different frequencies to two-hop neighbors, potential conflicts can not be avoided. Moreover, we note that MMSN's schemes allocate channels in static way which limits channel utilities as a node does not permanently use its assigned channel. In [5] and [6], the authors proposed a dynamic channel allocation based on agreement established between each sender and receiver nodes. Such approach may be suitable in light network but in dense network frequency negotiation messages can involve a considerable unnecessary overhead (e.g negotiation message retransmissions following their reception failure). The advantage of those protocols is the use of several channels for control traffic which can avoid control channel congestion problem. All previous multi-channel allocation schemes are proposed for classical WSNs operating on $2.4 \mathrm{Ghz}$ band without any support of quality-of-service (QoS) mechanisms, however the authors in [7] proposed the first Multi-Channel MAC protocol (MCMAC) taking into account the notion of priority during channel allocation process inside a cluster. In [8], the authors proposed the first multi-channel scheme designed for UWB based IEEE 802.15.3 networks. Based on dynamic traffic demand, the proposed mechanism employs a distributed dynamic channel allocation algorithm (DCA) [9] to distribute the channels among neighboring piconets. Due to several broadcasts (between PNCs (piconet controllers)), the communication overhead in this mechanism is relatively high. To support the channel allocation scheme, PNCs must be always active which represents an unacceptable condition on WSNs given the energetic constraints at WSN's nodes level. Then, inside each piconet, time slots are allocated based on MULTI-FIT [8] and Interval Based Scheduling (IBS) [8] algorithms to convert, in first step, the demand matrix into a specific form and to allocate time slots per available channels, in second step. Given that IBS algorithm is proposed to ensure time slot allocation for networks based on a star topology, resource allocation (Time slots per available channels) for Multi-Hop Wireless Mesh Networks can not be supported by this mechanism. Moreover, IBS does not provide any priority policy ensuring quality-of-service support. Similar to [8], we propose to organize the global network on set of PANs, to reduce the complexity of resource sharing. But, for channel and time slot allocation in UWB based WSNs, we propose a mechanism that obeys, on the one hand, to WSNs constraints and ensures, on the other hand, QoS support in such networks. The rest of the paper is organized as follows: In section 2, we present the system model. Section 3 details the proposed prioritized multi-channel and multi-time slot MAC protocol (PMCMTP). In section 4, we evaluate PMCMTP performance by analyzing and commenting some results.

\section{SYSTEM MODEL}

\section{A. IEEE 802.15.4a IR-UWB Spectrum Resource}

IEEE 802.15.4a IR UWB complaint devices can operate in three independent bands: (1) the sub-gigahertz band (250-750 $\mathrm{MHz})$, (2) the low band (3.1-5 GHz) and (3) the high band (6$10.6 \mathrm{GHz}$ ) (See Fig. 1). As shown in the table 39d given in [9], we dispose of 16 physical frequency channels associated with 8 sequence codes (i.e 32 logical channels).

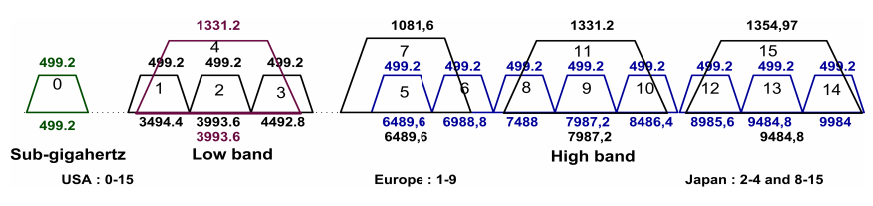

Fig. 1. IEEE 802.15.4a UWB plan bands

According to table 39d given in [10] and Fig. 1, neither overlapping channels nor adjacent channels share same sequence codes. Let us assume that $N_{t c h}$ represents the set of all available logical channels. Conforming to worldwide UWB spectrum regulation, $\operatorname{Card}\left(N_{t c h}\right)$ is equal to 32,18 and 22 for respectively US, Europe and Japan regions. Although bandwidths of the UWB channels are different, all proposed data rates ( 0.11 to $27.24 \mathrm{Mbps}$ ) can be supported by the IEEE 802.15.4a UWB physical layer operating with any channel.

The IEEE 802.15.4a MAC protocol supports two operational modes that can be selected by the PAN Coordinator:

- Beacon-enabled mode: The PAN Coordinator forces the use of a superframe structure (See Fig. 2) to manage communication between its network members.

- Non Beacon-enabled mode: There is no use of a superframe structure in this mode, the device can simply send their data by using unslotted CSAM/CA mechanism.

To provide time guarantees to deliver data frames, beaconenabled mode is used. The format of the superframe is defined by the PAN Coordinator. The superframe, corresponding to the Beacon Interval (BI), is defined by the time between two consecutive beacons, and includes an active period and, 
optionally, a following inactive period. The active period, corresponding to the Superframe Duration (SD), is divided into 16 equally sized time slots, during which data transmission is allowed. For a global network of $N_{c}$ PANs, each PAN coordinator is characterized by its superframe duration $\left\{P A N_{i}=\left(S D_{i}, B I_{i}\right)\right\}_{1 \leq i \leq N_{c}}$ as shown in Fig. 3.

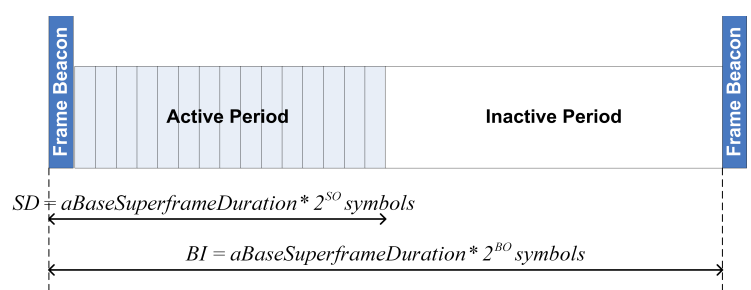

Fig. 2. PAN superframe structure

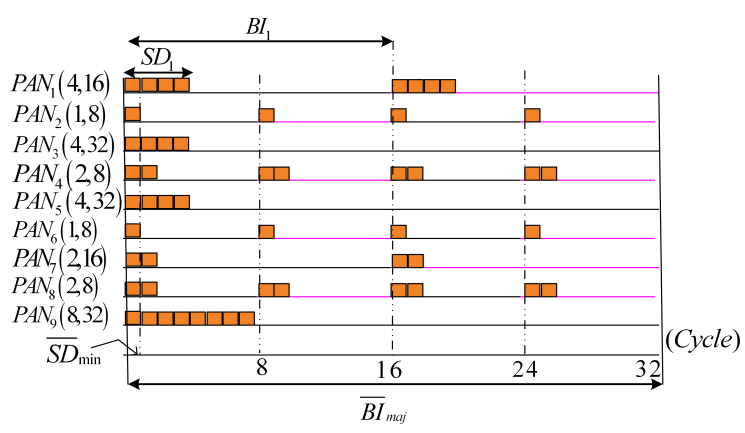

Fig. 3. Network configuration

During each elementary active cycle $\overline{S D}_{\text {min }}$, we can share available channels between only active PANs. Then, each active PAN coordinator must efficiently assign time slots per allocated channel to its PAN's members. According to radio transceiver characteristics, channel switch latency does not exceed 9.0ns (UWB devices) [8]. Although we can assume that during one duty cycle the additional delay introduced by radio channel switch is not significant, an efficient channelswitch scheme must be proposed to avoid unnecessary channel switches. To switch from a channel to another we just need to firstly program the set of available frequency channels at a specific register (e.g fsctrl.freq for CC2420 transceiver) then to select a given channel. This register must be set to the adequate value. Given the several advantages offered by the UWB technology, we propose to investigate the UWB physical layer specified by IEEE 802.15.4a standard for WSNs, by designing efficient schemes to ensure the best management of the UWB spectrum resource.

\section{B. Network Topology}

In order to deploy a dense network supporting a considerable number of nodes, we proposed in [11] a three-tiered network to represent the global network, using UWB sensors in the first and second network levels. We have chosen the UWB technology for the following raisons:
- its extremely low transmitting power minimizing interference and energy consumption,

- high data rates allowing real time and high data rate applications,

- location capacity ensuring mobility management and node identification.

For the third tier, we propose Wifi network to benefit from its high data rate, large coverage and security aspects. We aim to design a WHSN (Wireless Hospital Sensor Network) for an application in hospital (medical monitoring of patients and management of doctors). Fig. 4 shows all network layers composing a WHSN.

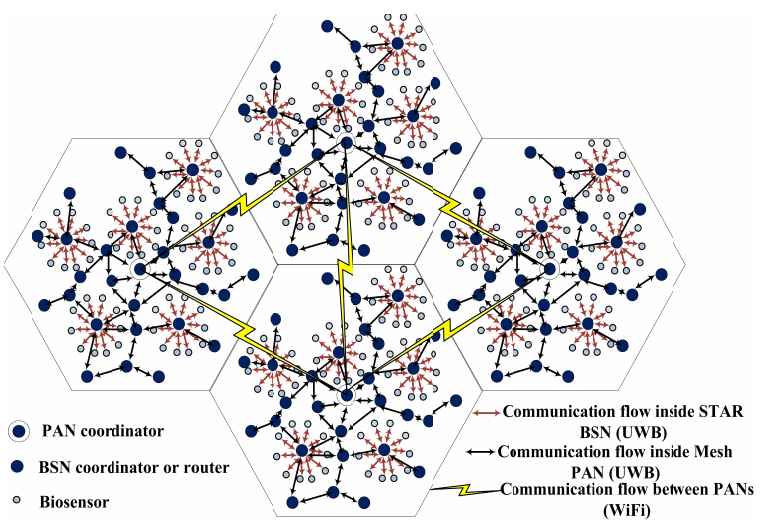

Fig. 4. WHSN architecture

The lowest level represents the Body Sensor Network (BSN). We can model an elementary BSN by a star network composed of one coordinator and a set of biosensors that ensure physiological measurements and the medical monitoring of patient. To improve patient's network performance in a dense hospital environment, we propose overlaying the network of BSNs with a second upper level network or PAN. As shown in Fig. 4, the network is represented by a hexagonal cell of sensors organized in a full mesh topology including one PAN's coordinator, several mobile BSNs coordinators (one coordinator per BSN) and several routers. For an efficient solution for channel allocation and mobility management in WHSNs, that cellular architecture, based on UWB/Wifi technologies, is chosen to the third level to have finally a three-tier hierarchical cellular network. The detailed description of the network architecture is out of scoop of this paper, so for more details, one can refer to [11]. Let us assume the general case of a network composed by $N_{c}$ PANs uniformly distributed. The ideal case of an hexagonal model is chosen to ensure the totality coverage of the network. Although in practice the coverage zone of a sensor device is neither an hexagon nor a perfect circle, there are procedures and mechanisms [12] that ensure the adjustments of the model during network deployment by means of experimental test of measurements. To benefit completely from advantages offered by the UWB technology, we treated, in [13], the problem of UWB-channels sharing between PANs and we propose, in the following section, a new MAC protocol ensuring an efficient 
multi-channel and multi-time slot sharing inside PANs.

\section{PMCMTP FOR AN IR UWB SENSOR NETWORK}

A WHSN is intended to ensure real time and continuous patient monitoring, to reduce time of routine consultation and to immediately treat emergency cases. So, such network must support a huge number of BSNs or patients with different states which must be monitored by means of various types of biosensors. Consequently, the network must support QoS ensuring efficient patient monitoring. In order to enhance such QoS in terms of network capacity or throughput increase, delays decrease, prioritized physiological measurements support, load and energy balancing, we propose a PMCMTP for mesh WSN taking into account:

- Spatial channel reuse in order to efficiently assign several channels per PAN without suffering from co-channel interference,

- PANs duty cycle in order to dynamically allocate channels and maximize channel utility,

- Data stream prioritization at the level of PANs and BSNs, to ensure QoS support per patient and per service.

\section{A. First Level of Channels Allocation}

Inside a WSN, we distingue two types of traffic: Control and data communication traffics. Control traffic is generated to identify each PAN, to synchronize devices that are associated with a PAN and to manage communication between each PAN's members. Data communication traffic represents the useful information to be transmitted between devices.

1) Control channel allocation: To avoid control channel congestion [6], we propose to statically assign one control channel to each PAN. The emission power density of the UWB signals is less than $-41.3 \mathrm{dBm} / \mathrm{MHz}$ [10]. Given that, overlapping channels $(4,7,11$ and 15) are characterized by high bandwidth [10], they can allow higher transmit power, permitting an extended range, compared to non-overlapping channels. To persistently cover each cell with control traffic, we find that the overlapping channels are more suitable to ensure the zone coverage for such traffic.

2) Dynamic data communication channel allocation: According to network configuration and by means of an optimal coloring algorithm, we propose to allocate the set of residual channels (non-overlapping channels and the supplementary overlapping channels with their appropriate sequence codes) for data communication. According to PAN's duty cycle and available channel frequencies, each PAN coordinator can benefit simultaneously from several data communication channels. The detailed description of UWB-channels (Control and data channels) sharing between PANs is out of scoop of this paper, so for more details, one can refer to [13].

\section{B. Second Level of Data Channels Allocation (inside a PAN)}

In this subsection, we propose a method of logical channels and time slots allocation inside each PAN. For each cycle, PAN coordinator collects all the resource allocation requests of its network's members. Then, according to the spectrum sharing scheme [11], it can know the number of channels that it can benefit from during the current active cycle. Next, it tries to allocate available time slots per channel in response to collected requests. Finally, concerned sensors can begin their data communications. The principle of proposed protocol is based on the three following phases:

1) Phase for requesting time slots,

2) Phase of channels/time slots allocation,

3) Phase of data transmission.

- Phase for requesting time slots: Compared to MCMAC protocol [7], PMCMTP begins with a phase of requests transmission but it takes into account not only channel allocation task but also a dynamic time slots assignment. On the one hand, in PMCMTP, the number of requested time slots can vary from one transmission request to another according to the application needs. On the other hand, we distinguish two types of request: BSN transmission request (communication inside $\mathrm{BSN}$ ) and routing transmission request (communication between PAN's members). Transmission requests phase must precede each PAN's active period. As shown in Fig. 5, this phase is divided into two sub steps, in order to synchronize the PAN in the first step, and to collect all transmission requests of concerned PAN's members, in the second step. During this phase, the allocated control channel is used. During the first step, by listening to the beacon frame, PAN members adjust their wake-up clocks. The second step represents a set of equal short time slots, during which, the PAN coordinator is listening to the requests of PAN's member. So, inside each PAN, according to the number of members, PAN coordinator assigns to each member a specific time slot according to its ID. Earlier time slot is assigned for lower ID. As shown in Fig. 5, just following the reception of the first beacon frame, each PAN's member waits for its own time slot in order to send its request packets.

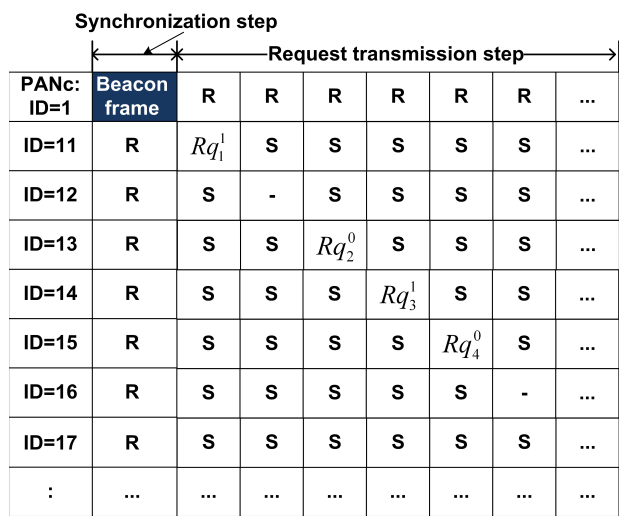

Fig. 5. Phase of transmission requests

The request packet is composed of 7 fields (Fig. 6):

- Type: Type of packet,

- $R q_{I D}$ : Request Identifier, 
- $R q_{\text {type }}$ : Type of resource allocation request:

* $R q^{0}$ : For communication inside BSN,

* $R q^{1}$ : For routing communication inside a PAN.

- P: Request's priority level,

- TS: Number of required time slots,

- @Source: @ of the request's sender,

- @Destination: @ of flow destination.
The RSA algorithm can be better explained by following an example. Considering the case of four resource requests

\begin{tabular}{l}
\hline Notation \\
\hline$R Q:$ Set of requests, $R q_{\text {First }}:$ A temporary set of requests \\
$R q_{S e r v e d}:$ Set of served requests, $R q_{\text {inqueue }}:$ Set of requests in queue \\
$R q_{i}^{k}:$ A resource request where $k \in\{0,1\}, P_{i}:$ Priority Level of a request \\
$F T S_{i}:$ First time slot, $L T S_{i}:$ Last time slot, $T S_{i}:$ Time slots \\
$I D_{i}:$ Identifier of request's sender, $S_{i}:$ Address of the request's sender \\
$@ D_{i}:$ Address of flow destination, $A T S_{i}:$ First allocated time slot, \\
$A C H_{i}:$ Allocated channel, $N_{i}:$ Number of hops, $T S / C H:$ TS per channels \\
\hline
\end{tabular}

Algorithm 1 Request Scheduling Algorithm (RSA)

1. Collection of resource requests $R q_{i}^{k}$

$R Q=\left\{R q_{i}^{k}, k \in\{0,1\}\right\} \cup R q_{\text {inqueue }}$

$\forall R q_{i}^{k} \in R Q$ Set $F T S_{i}=0$ and $L T S_{i}=T S_{i}$

2. Decomposition of each routing resource request $R q_{i}^{1}$

into a set of $N_{i}$ routing resource requests of one hop where:

Set $F T S_{i}^{1}=F T S_{i}$ and $L T S_{i}^{1}=L T S_{i}$

Set $F T S_{i}^{j}=L T S_{i}^{j-1}$ and $L T S_{i}^{j}=F T S_{i}^{j}+T S_{i}, j \in\left[2, N_{i}\right]$

$j$ refers to the $j^{\text {th }}$ hop in the path

3. Selection of more priority resource requests: $R q_{\text {First }}$

2.a. Set $R q_{\text {First }}=$

$\left\{R q_{i}^{k} \in R Q\right.$ where $\left.P_{i}=\min \left(P_{j}\right), \forall R q_{j}^{k} \in R Q\right\}$

2.b. Set $R Q=R Q-R q_{\text {First }}$

4. Conflict avoidance between $R q_{i}^{k}$ with same priority

If $\exists\left(R q_{i}^{k}, R q_{j}^{k}\right) \in R q_{\text {First }}$ where:

$\left[\left(@ S_{i}=@ D_{j}\right)\right.$ or $\left.\left(@ D_{i}=@ D_{j}\right)\right]$ and

$\left[\left[F T S_{i}, L T S_{i}\right] \cap\left[F T S_{j}, L T S_{j}\right] \neq \emptyset\right]$ Then

If $\left(T S_{i}<T S_{j}\right)$ or $\left(\left(T S_{i}=T S_{j}\right)\right.$ and $\left.\left(I D_{i}<I D_{j}\right)\right)$ Then

Set $F T S_{j}=L T S_{i}, L T S_{j}=F T S_{j}+T S_{j}$

5. Conflict avoidance between $R q_{i}^{k}$ with different priority

If $\exists\left(R q_{i}^{k}, R q_{j}^{k}\right) \in R q_{\text {First }} \cup R Q$ where:

$\left[\left(@ S_{i}=@ D_{j}\right)\right.$ or $\left.\left(@ D_{i}=@ D_{j}\right)\right]$ and

$\left[\left[F T S_{i}, L T S_{i}\right] \cap\left[F T S_{j}, L T S_{j}\right] \neq \emptyset\right]$ Then

If $\left(P_{i}<P_{j}\right)$ Then

Set $F T S_{j}=L T S_{i}, L T S_{j}=F T S_{j}+T S_{j}$

6. Schedule $R q_{\text {First }}$ where:

If $\left(F T S_{i}<F T S_{j}\right)$ Then Set $R q_{j}^{k}$ after $R q_{i}^{k}$

Else If $\left(F T S_{i}>F T S_{j}\right)$ Then Set $R q_{i}^{k}$ after $R q_{j}^{k}$

Else If $\left(T S_{i}<T S_{j}\right)$ or $\left(T S_{i}=T S_{j}\right.$ and $\left.I D_{i}<I D_{j}\right)$ Then Set $R q_{j}^{k}$ after $R q_{i}^{k}$

7. Allocation of time slots per available channel

while $(R Q \neq \emptyset$ and $T S / C H$ are available $)\{$

7.a while $\left(R q_{\text {First }} \neq \emptyset\right.$ and $T S / C H$ are available $)\{$

$\forall R q_{i}^{k} \in R q_{\text {First }}$

$A T S_{i}=$ lowest available Time slot $\geq F T S_{i}$,

$A C H_{i}=$ index of relative channel

$R q_{\text {First }}=R q_{\text {First }}-R q_{i}^{k}$

$\left.R q_{\text {Served }}=R q_{\text {Served }} \cup R q_{i}^{k}\right\}$ End While

7.b repeat $2,3,4,5$ and 6$\}$ End While

8. If $R Q \neq \emptyset$ Then $R q_{\text {inqueue }}=R Q$ over the accorded control channel using the mandatory data rate $(850 \mathrm{Kbps})$. During data communication phase, sensors communicate (T: Transmission, R: Reception, and BSN: BSN's communication) using the accorded data communication channels.
(TABLE I) and three channels 0,1 and 2 (represented by blue, green and purple colors), after the processing of received 
requests, RSA allocates required time slots per available channels to each request as shown in TABLE II. Fig. 8 illustrates synchronization and data transmission phases.

TABLE I

EXAMPLE OF FOUR RESOURCE REQUESTS

\begin{tabular}{|c|c|c|c|c|c|c|}
\hline$R q_{I D}$ & $R q_{\text {type }}$ & $\mathrm{P}$ & $\mathrm{TS}$ & Source & Destination & Path \\
\hline \hline 1 & 1 & 1 & 2 & 11 & 13 & $11 \rightarrow 12 \rightarrow 13$ \\
\hline 2 & 1 & 2 & 1 & 14 & 11 & $14 \rightarrow 11$ \\
\hline 3 & 0 & 4 & 4 & 15 & 15 & - \\
\hline 4 & 0 & 3 & 2 & 13 & 13 & - \\
\hline
\end{tabular}

TABLE II

RESOURCE ALLOCATION OF THE PREVIOUS EXAMPLE

\begin{tabular}{|c|c|c|c|c|c|}
\hline$R q_{I D}$ & ACH & FTS & ATS & Source & Destination \\
\hline \hline 1 & 0 & 0 & 2 & 11 & 12 \\
\hline 1 & 0 & 2 & 2 & 12 & 13 \\
\hline 2 & 1 & 2 & 1 & 14 & 11 \\
\hline 3 & 1 & 0 & 2 & 13 & 13 \\
\hline 4 & 2 & 0 & 4 & 15 & 15 \\
\hline
\end{tabular}

\begin{tabular}{|c|c|c|c|c|c|c|c|c|}
\hline $\begin{array}{l}\text { PANc: } \\
\text { ID =1 }\end{array}$ & $\begin{array}{l}\text { Beacon } \\
\text { frame }\end{array}$ & & & & & & & $\ldots$ \\
\hline$I D=11$ & $\mathbf{R}$ & $T$ & $T$ & $\mathbf{R}$ & & & & $\ldots$ \\
\hline$I D=12$ & $\mathbf{R}$ & $\mathrm{R}$ & $R$ & $T$ & $T$ & & & $\ldots$ \\
\hline $\mathrm{ID}=13$ & $\mathbf{R}$ & & & $\mathbf{R}$ & $\mathbf{R}$ & & & $\ldots$ \\
\hline $\mathrm{ID}=14$ & $\mathbf{R}$ & & & $T$ & & & & $\ldots$ \\
\hline$I D=15$ & $\mathbf{R}$ & & & & & & & $\ldots$ \\
\hline $\mid D=16$ & $\mathrm{R}$ & & & & & & & $\ldots$ \\
\hline$I D=17$ & $\mathbf{R}$ & & & & & & & $\ldots$ \\
\hline : & $\ldots$ & $\ldots$ & $\ldots$ & $\ldots$ & $\ldots$ & $\ldots$ & $\ldots$ & $\ldots$ \\
\hline
\end{tabular}

Fig. 8. Reception of beacon frame and beginning of data transmission

\section{Performance evaluation}

We have implemented PMCMTP in a discrete-time simulator built in JAVA and based on some functionalities defined by Prowler simulator [14] with the support of the network architecture proposed in [11] and the spectrum sharing scheme detailed in [13]. In this section, we propose to evaluate the performance of PMCMTP.

\section{A. Simulation Parameters}

Let us consider a synchronized network of 9 hexagonal cells of radius $R=5 \mathrm{~m}$. Each PAN has 35 nodes uniformly distributed (One PAN coordinator, thirty routers and four BSNs coordinators). Each PAN coordinator is characterized by its superframe duration $\left\{P A N_{i}=\left(S D_{i}, B I_{i}\right)\right\}_{1<i<9}$ as shown in Fig. 2 and Fig. 3. We define $\overline{B I_{m a j}}$ and $\overline{S D_{\min }}$ as respectively the major cycle and the elementary active cycle.

$$
\begin{aligned}
& \overline{B I_{\text {maj }}}=\operatorname{LCM}\left(B I_{1}, B I_{2}, \ldots, B I_{9}\right)=\max _{1 \leq i \leq 9}\left(2^{B O_{i}}\right) \\
& \overline{S D_{\text {min }}}=L C D\left(S D_{1}, S D_{2}, \ldots, S D_{9}\right)=\min _{1 \leq i \leq 9}\left(2^{S O_{i}}\right)
\end{aligned}
$$

So, $\overline{B I_{\operatorname{maj}}}=32, \overline{S D_{\min }}=1$.

TABLE III shows the default value of each parameter in the simulations. To eliminate the bottleneck problem of single sink node, we assume that there are several sink nodes in each PAN.

TABLE III

\begin{tabular}{|c|c|}
\hline Parameter & Default value \\
\hline Number of PANs, Nodes per PAN & 9,35 \\
\hline Communication rate & $850 K b p s$ \\
\hline Radio range & $5 m$ for control, $2 m$ for data \\
\hline Data Packet length & 127Bytes \\
\hline $\begin{array}{c}\text { System Load } \\
\text { per elementary active cycle }\end{array}$ & $\begin{array}{l}\text { Variable according to the number and } \\
\text { characteristics of resource requests }\end{array}$ \\
\hline Characteristic of resource requests & $P \in\{1-5\} T S \in\{1-4\}$ \\
\hline Time slot duration & $0.985 \mathrm{~ms}$ \\
\hline$S O_{\min }$ & $\begin{array}{l}4,(P A N \text { 's superframe Order } \\
\left.\text { will be normalized by } S O_{\min }\right)\end{array}$ \\
\hline Routing Laye & Geographic-Based Shortest Path Routing \\
\hline
\end{tabular}

SiMULATION PARAMETER

\section{B. Network Channel Allocation per Active Cycle}

According to the network's configuration shown in Fig. 3 and channel allocation scheme detailed in [12] each PAN during its active elementary cycle can benefit from several channels of frequencies. As shown in Fig. 9 during the $1^{\text {st }}$, $2^{\text {nd }}$ and $17^{\text {th }}$ elementary cycles each active PAN allows up to 4, 6 and 9 simultaneous communications respectively with European, Japan and US spectrum regulation. Where during the $3^{\text {rd }}, 4^{\text {th }}, 9^{\text {th }}, 18^{\text {th }}$ and $25^{\text {th }}$ elementary cycles, each active PAN benefits from 7, 9 and 14 channels respectively with European, Japan and US regulation. For a load of $10 \mathrm{CBR}$ per elementary active cycle per PAN, we note that each active PAN is able to simultaneously support all communications with Japan and US UWB spectrum regulation given that the number of available channels is bigger than supported data communications. During the $5^{\text {th }}, 6^{\text {th }}, 7^{\text {th }}, 8^{\text {th }}, 10^{\text {th }}$, $19^{t h}, 20^{t h}$ and $26^{t h}$ elementary cycles, active PANs benefit simultaneously from all available channels and all independent communications are simultaneously served.

\section{Delay Evaluation}

1) Required time slots vs channel and system loads variety: We take the number of required time slots to serve a set of resource requests $(5,10$ and 15 resource requests per PAN) as a metric to measure PMCMTP performance. To analyze performance scalability, we conduct simulation with different numbers of channel frequencies as well. Fig. 10 shows that, with all system loads, our protocol becomes more time efficient when we increase the number of channel frequencies. For this reason at the level of the global network, we impose a dynamic data channel allocation based on PANs duty cycle's information and spatial frequency reuse to avoid the underutilization of spectrum resource and to maximize the number of used channels per active PAN. We note that the amount of required time slots in the case of 5, 10 and 15 resource requests decreases respectively by $3.5,8$ and 12.25 times when the number of channels increases from 1 to 15 . 
2) End-to-End delay vs channel variety: We define the average end-to-end delay per packet as the ratio of the sum of delay $\left(\delta_{i}\right)$ experienced by each packet making up the flow per number of packets $(N)$.

$$
A V G(\text { End2End Delay per packet })=\frac{\sum_{i=0}^{N} \delta_{i}}{N}
$$

Fig. 11 highlights the PMCMTP's behavior in terms of the average end-to-end delay per packet through the whole network when different numbers of channels are used. For a load of 15 resource requests per active PAN and a data rate of $850 \mathrm{Kbps}$, we note that the $A V G$ End-to-End delay per packet decreases when the number of used channels increases more and it can be reduced respectively by $562 \%$ and $100 \%$ when the number of channels increases from 1 to 8 and from 8 to 15 .

3) End-to-End delay vs data rate variety: We explore PMCMTPs performance when different data rates are used for the same amount of load. As shown in Fig. 11, for all different data rates, it is observed that PMCMTP always exhibits better performance when more channels are used, which is consistent with the result presented in the previous experiments. Also, we note that the $A V G$ End-to-End delay per packet decrease slows down when data rate increases more given that delay experienced by packet decreases with data rate increase.

4) Max End-to-End delay vs node density variety: We propose to evaluate the effect of node density variation on PMCMTP's behavior by configuring the network with different cell's radius varying from 3 to $8 \mathrm{~m}$ with the same number of nodes and system load (10 random resource requests per PAN). We also explore the difference of performance with different numbers of channels as well. Fig. 12 shows that PMCMTP always exhibits better performance in terms of end to end delay with smaller radius size (i.e with higher node density). We can explain the increase of the end to end delay when cell's radius size increases, on one hand, by the increase of the number of hops per route and, on the other hand, by the phenomena of congestion at some intermediate nodes.

\section{Throughput Evaluation}

1) Throughput vs time and channel variety: Taking the network throughput as metric, we analyze the behavior of PMCMTP and MCMAC [7] as a function of time. We conduct experiment with 10 random resource requests per PAN. According to the network configuration, the number of active PANs and the number of used channels per PAN can vary from elementary cycle to another. Fig. 13 shows that the data throughput increases with the increase of the number of active PANs (which leads to the increase of the number of source nodes) and the number of used channels because more nodes get involved in the communication and more parallel data transmissions occur. We note that, when the number of channels decreases below certain value, the phenomena of congestion appears and the increase of throughput slows down. Compared to MCMAC, PMCMTP exhibits better performance given that MCMAC allows only one PAN to be active and it allocates data channels for a fixed duration which may introduce additional communication overhead. Fig. 14 shows the evolution of the throughput of the global network as a function of the number of used channels with a load of 15 random resource requests per active PAN. We note that the data throughput increases when channel increases more. Once again, our results confirm that PMCMTP always achieves a higher performance when more channels are available.

2) Throughput vs node density variety: Fig. 15 shows that the data throughput increases with the decrease of node density, especially when the number of channels is important. This is due, on the one hand, to the increase of the number of hops per route and, on other hand, to the increase of parallel transmissions. For a trade-off between throughput and end to end delay, we must choose a rational node density value.

\section{CONClusion}

In this paper, we propose a novel prioritized multi-channel multi-slot MAC protocol for dense and large-scale wireless sensor network, which is decomposed into a set of PANs organized in full mesh topology in order to reduce the complexity of resource sharing task and to balance load and energy consumption. The support of QoS is the primary goal in the protocol design. So, according to the network configuration and the available spectrum resource, PMCMTP tries to efficiently assign time slots per channel in response to received resource requests. We evaluate PMCMTP's performance through a set of simulations, and the experimental results show that our protocol exhibits prominent ability to utilize parallel transmissions without suffering from interference, data communication conflict and control packet overhead. PMCMTP also achieves a considerable enhancement of the performance of the global network when multiple channels of frequencies are available. In future, we will study energy consumption in Multi-channel MAC layer and evaluate the impact of other layers on the proposed protocol.

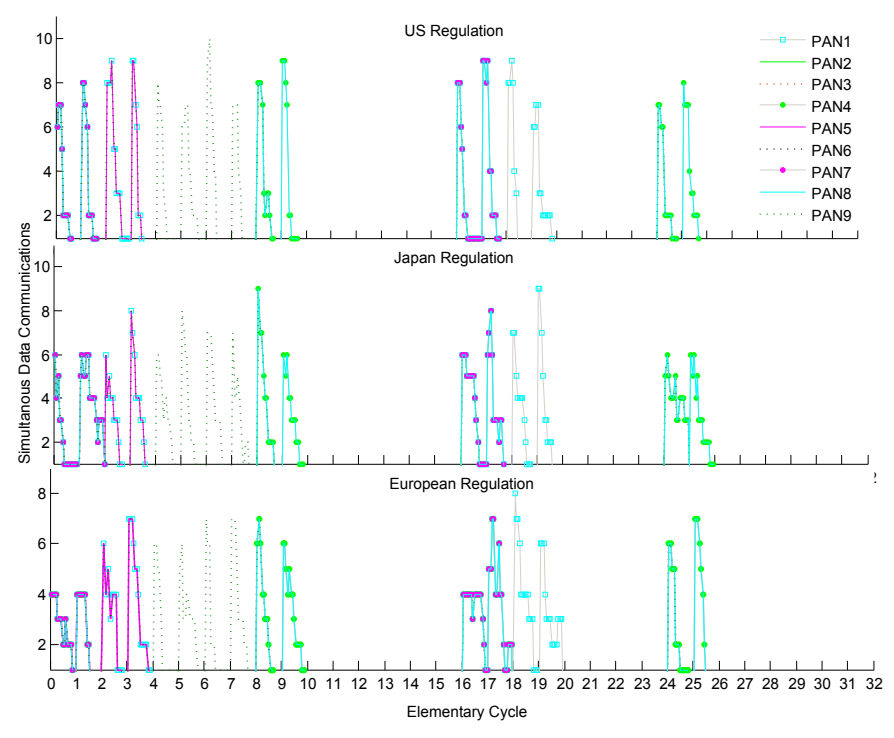

Fig. 9. Channel Allocation per active cycle vs spectrum Regulations 


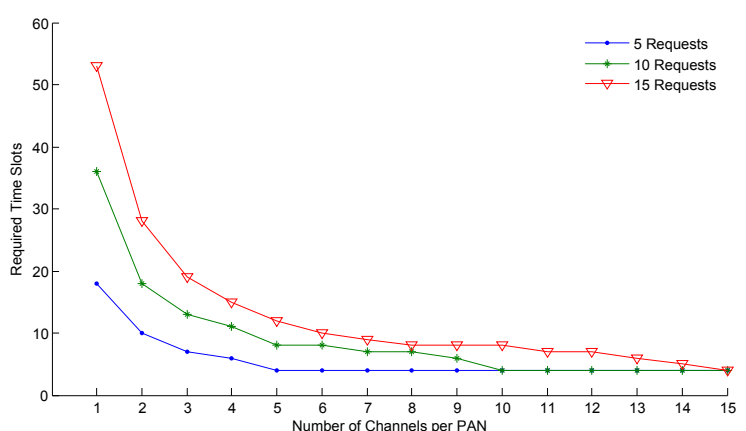

Fig. 10. Required Time Slots vs Channel Variety

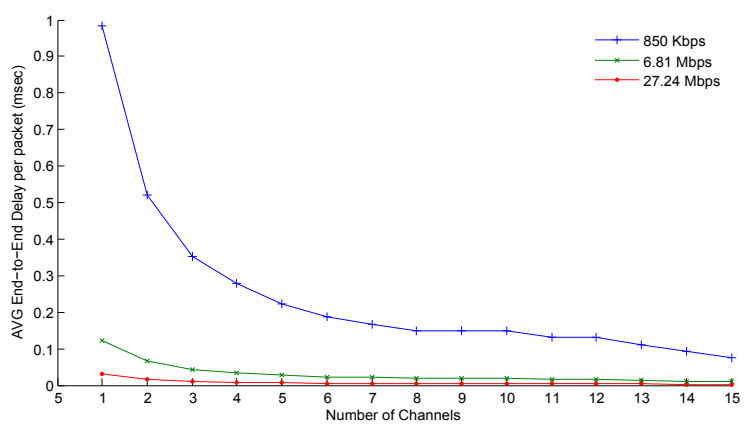

Fig. 11. AVG End-to-End Delay per PAN vs Channel and Data rate Variety

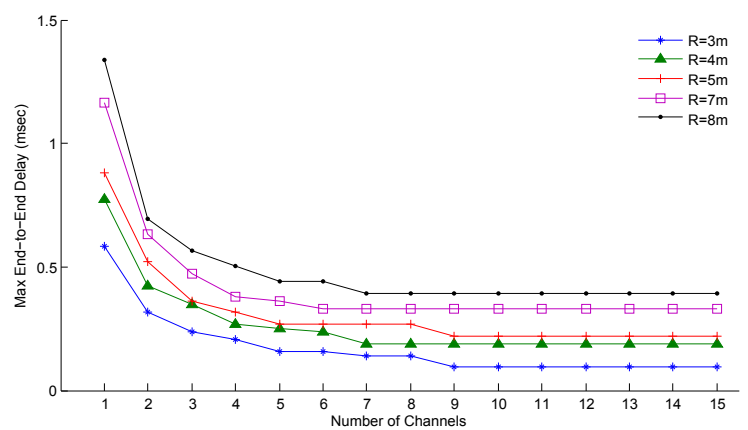

Fig. 12. Max End-to-End Delay vs Node Density Variety

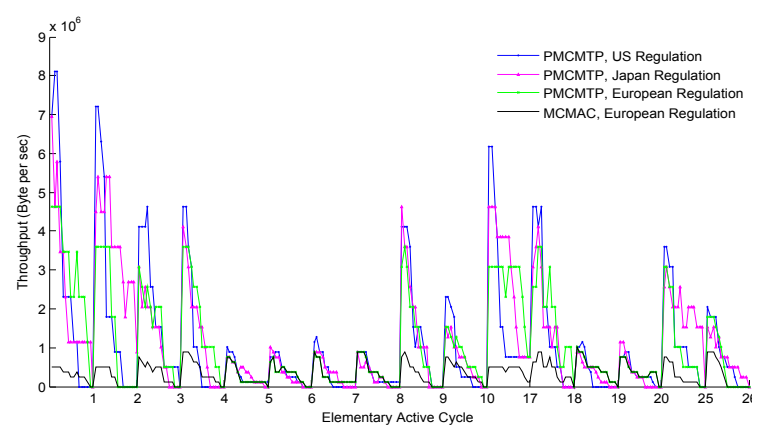

Fig. 13. Throughput vs Time

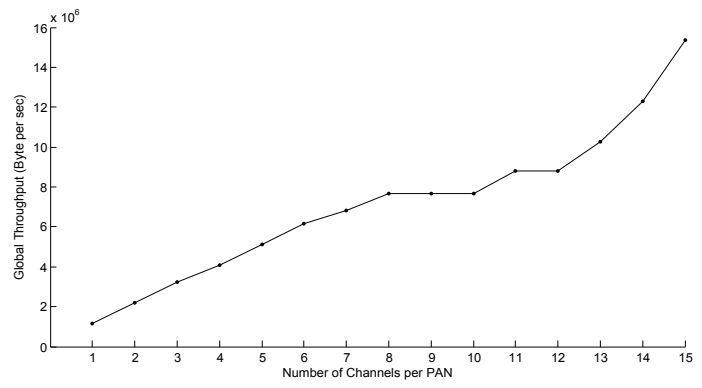

Fig. 14. Throughput vs Channel Variety

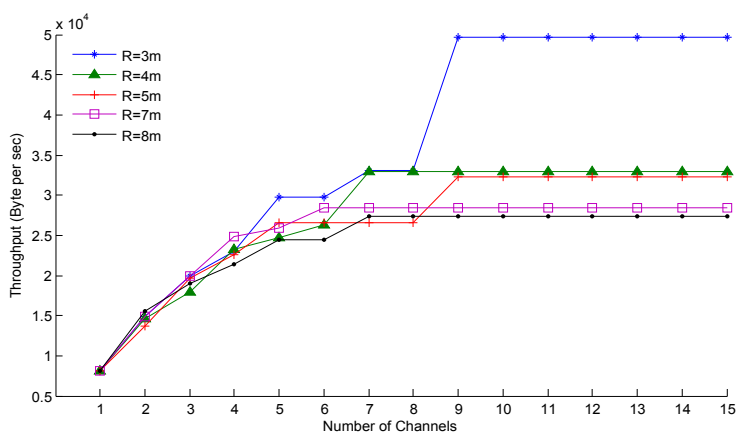

Fig. 15. Throughput vs Node density Variety

\section{REFERENCES}

[1] X. Wang and T. Berger, "Spatial channel reuse in wireless sensor networks", Springer, vol 14, iss 2, pp. 133-146, March 2008.

[2] CC2420 datasheet, inst.eecs.berkeley.edu/ cs150/Documents/CC2420.pdf

[3] X. Lagrange, P. Godlewski and S. Tabbane, "Reseaux GSM-DCS ", Herms Sciences Publications.

[4] G. Zhou, C. Huang, T. Yan, T. He and J. Stankovic, A. Abdelzaher, "MMSN: multi-frequency media access control for wireless sensor networks", INFOCOM 2006. 25th IEEE International Conference on Computer Communications Proceedings, April 2006, pp. 1 - 13.

[5] R.E. Cagley, S.A. McNally and M.R. Wiatt, "Dynamic channel allocation for dynamic spectrum use in wireless sensor networks" Military Communications Conference, October 2006, pp. 1-5.

[6] H. So, W. Walrand and J. Jeonghoon, "McMAC: a parallel rendezvous multi-channel MAC protocol", IEEE Wireless Communications and Networking Conference, March 2007, pp. 334-339.

[7] X. Chen, P. Han, Q. He, S. Tu and Z. Chen,'A multi-channel MAC protocol for wireless sensor networks", The 16th IEEE International Conference on Computer and Information, 2006, pp.224-229.

[8] A. Rangnekar, K.M. Sivalingam, "Multiple Channel Scheduling in UWB based IEEE 802.15.3 Networks", Proceedings of the First International Conference on Broadband Networks, October 2004, pp.406-415.

[9] R. Prakash, N. Shivaratri, and M. Singhal, "Distributed Dynamic Channel Allocation for Mobile Computing", In Proceedings of the 14 th ACM Symposium on Principles of Distributed Computing, August 1995.

[10] IEEE 802.15.4a Standard Part 15.4: IEEE Standard for Information Technology, Amendment to IEEE Std 802.15.4-2006, 2007.

[11] J. Ben Slimane, Y.Q .Song, A. Koubaa, M. Frikha, "A Three-Tiered Architecture for Large-Scale Wireless Hospital Sensor Networks", the International Workshop on Mobilizing Health Information to Support Healthcare-Related Knowledge Work - MobiHealthInf 2009, pp 20-31.

[12] J. Jemai, R. Piesiewicz, T. Kurner, "Calibration of an indoor radio propagation prediction model at $2.4 \mathrm{GHz}$ by measurements of the IEEE $802.11 \mathrm{~b}$ preamble" IEEE 61st Vehicular Technology Conference, Spring. 2005, Vol 1, pp.111-115.

[13] J. Ben Slimane, Y.Q .Song, A. Koubaa, M. Frikha, "Allocation of control and data channels for Large-Scale Wireless Sensor Networks", Technical report, 2008, http://hal.inria.fr/inria-00322584/fr/.

[14] http://www.isis.vanderbilt.edu/projects/nest/prowler/. 